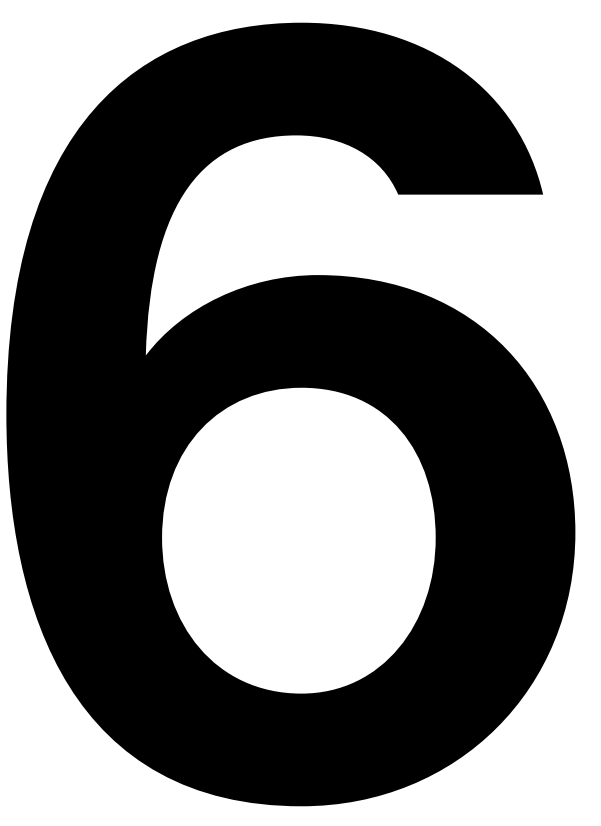

\title{
Videos analysieren: Verortungen und Annotationen Gunter Lösel
}

\author{
6.1 Verortung des eigenen Projekts \\ 6.2 Die Analyse mit Videos und Annotationen
}


In der künstlerischen Forschung ist der Bezug zu einer bestimmten Forschungstradition nicht von vornherein gegeben und kann daher auch nicht vorausgesetzt werden. Wie können sich Forschende zu Beginn eines Forschungsvorhabens unter Verwendung von Video und Annotation in der Landschaft der Forschungsmethoden verorten? Dieses Kapitel zeigt Wege auf, wie die Analyse eines Videos auf relevanten Entscheidungen basiert werden kann und damit in sich konsistent bleibt. 
Die Analyse von Videos ist nie ganz voraussetzungslos, und ein erster Schritt besteht darin, dass die Forschenden sich im Feld möglicher Forschungsansätze verorten und dadurch ihre eigenen Grundannahmen reflektieren können. René Tuma legte 2018 einen Überblick über die wichtigsten Forschungstraditionen vor, die im Kontext von Videoanalysen wichtig sind:

Diese Einteilung $(\rightarrow$ Abb. 01) verdeutlicht die historische Gewachsenheit der Ansätze, fragt also nach ihrer Einbettung in bestehende Theorien. Gemäß der Zielsetzung unseres Projekts wurde versucht, diese disziplinäre Gebundenheit zu überwinden.

\subsection{Verortung des eigenen Projekts}

Zielt meine Forschung auf manifeste oder latente Muster?

Die Ansätze lassen sich danach einteilen, ob sie eher die Oberfläche oder die Tiefenstruktur der Daten untersuchen. Gubrium und Holstein beschreiben dies als das Zusammenspiel der »Whats « - dem konkreten, beschreibbaren Inhalt - mit den »Hows « - den Zusammenhängen, die nicht direkt beobachtbar sind und daher erschlossen werden müssen (Gubrium/Holstein 1997). Entsprechend kann man die verschiedenen Ansätze auch dahingehend einordnen, ob sie eher das »Was« oder das »Wie« des Materials untersuchen:

Diese Entscheidung $\rightarrow$ Abb. 02) bestimmt unter anderem, welche und wie viele Daten erhoben werden: Während die Suche nach manifesten Mustern in der Regel eine breite Datenbasis benötigt, ist beim Fokus auf latente Muster eine Beschränkung auf kleine, aber gut ausgewählte Datenmengen üblich. Mit der Entscheidung »manifest vs. latent « legt man also auch fest, ob man mehr in die Breite oder mehr in die Tiefe forschen will.

Beispielsweise werden in der künstlerischen Forschung oft Prozesse untersucht, die nicht bewusst und deklarativ sind. Vielmehr handelt es sich um »tacit knowledge«, implizites Wissen, das erst durch spezielle Verfahren beobachtbar und beschreibbar gemacht werden muss. Künstlerische Forschung zielt damit oft auf latente Strukturen (jedoch geht es im Gegensatz zu den anderen hermeneutischen Methoden nicht um die Rekonstruktion von latenten Sinnstrukturen, sondern um eine Fülle von intuitiven, körperbezogenen, materialbezogenen und anderen Verfahren, die »irgendwie« zu einem künstlerisch relevanten Output führen). Die entsprechende Forschung versucht, diese unsichtbaren 


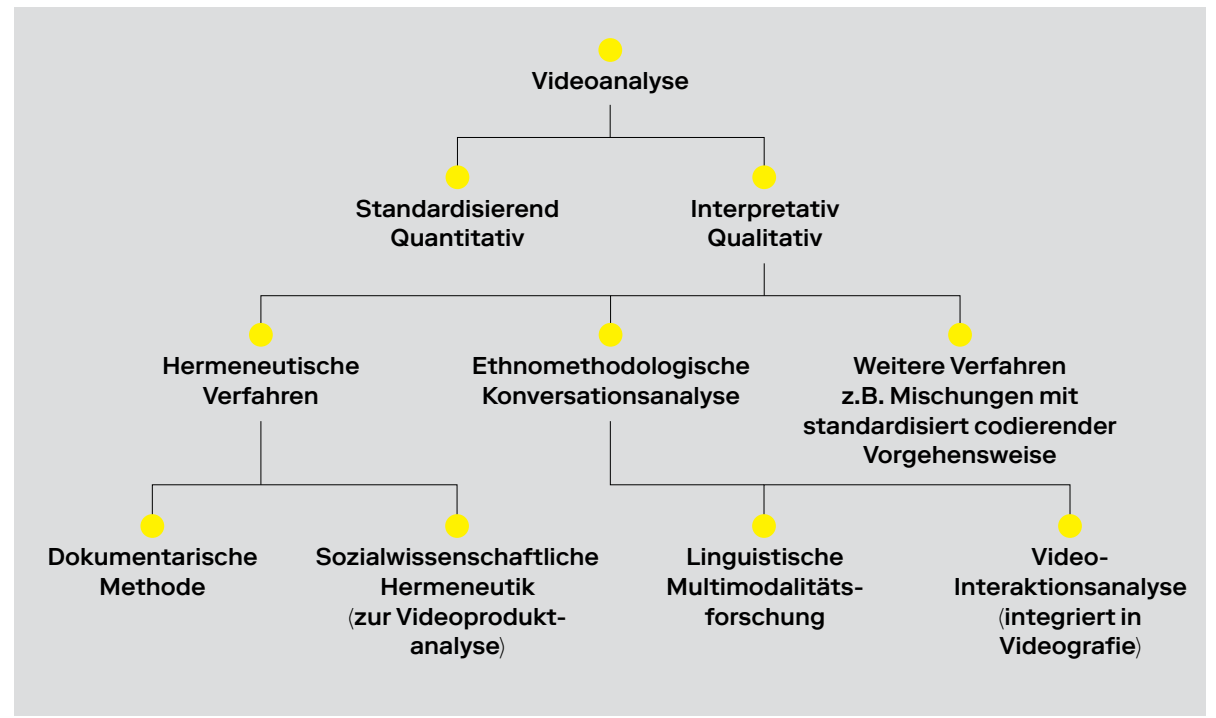

\section{1}

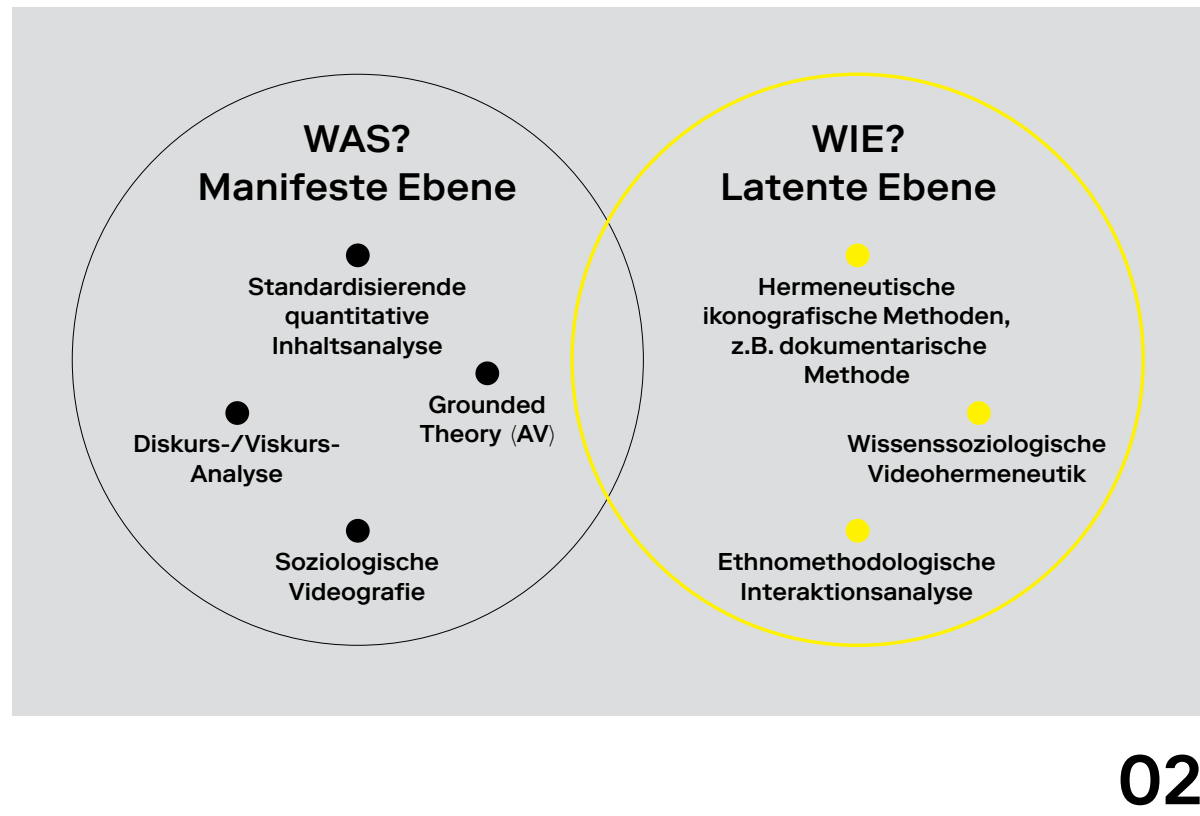

Abb. 01:

Verfahren der Videoanalyse nach

René Tuma (Quelle: Tuma 2018, S. 426)

Abb. 02:

»Was« und »Wie« der Analyse

(Quelle: eigene Darstellung) 
Strukturen und verkörperten Praktiken sichtbar zu machen. In anderen Fällen bezieht sich die künstlerische Forschung aber auch auf das explizite, deklarative Wissen der Künstler, das in Interviews oder Video-Cued-Recall-Situationen abgefragt werden kann. In solchen Fällen zielt sie auf manifeste Inhalte oder »deklaratives Wissen«.

Welche Teile des Videomaterials sollen untersucht werden?

Eine radikale Antwort ist: alles, was aufgenommen wurde - und aufgenommen werden sollte so viel wie möglich. Diesen Anspruch erhebt beispielsweise die visuelle Grounded Theory/Mey/Dietrich 2016). Ebenfalls auf das gesamte aufgenommene Material bezieht sich die dokumentarische Methode (Burkhard 2019): Hier geht es durchaus um große Datenmengen, denn die gesuchten Muster (z. B. der individuelle Stil des Autors/der Autorin) findet sich nicht hier und dort, sondern überall im Material.

Gewöhnlich wird aber eine Selektion vorgenommen, das heißt, nur ausgewählte Schlüsselstellen werden untersucht. Dies trifft auf alle hermeneutischen Verfahren zu. Die Auswahl dieser Stellen, das sogenannte Sampling, wird damit zu einem entscheidenden Vorgang, der methodisch gut begründet werden muss.

In extremen Fällen werden nur winzige Teile des Videos herangezogen (eventuell nur einzelne Stills). Dies gilt beispielsweise für Verfahren, die sich aus der Bildanalyse herleiten, zum Beispiel die figurative Hermeneutik (Eisewicht et al. 2018), wo durchaus mit Einzelbildern gearbeitet werden kann. Auch die objektive Hermeneutik nach Ulrich Oevermann kann mit sehr wenig Datenmaterial auskommen (Oevermann et al. 1979).

Welchen Kontext ziehe ich zur Interpretation heran?

Forschende können sich einer Frage oder einem Phänomen mit sehr viel Vorwissen nähern oder versuchen, es aus sich selbst heraus zu verstehen. Die verschiedenen Forschungsansätze gehen hier unterschiedlich vor:

Kein Kontext. Die Äußerung wird unter der Maßgabe der Kontextfreiheit untersucht (z. B. in der objektiven Hermeneutik). Dies ermöglicht, unvoreingenommen an das Sample heranzugehen und auch ungewöhnliche Interpretationen zu aktivieren. Daher muss der Kontext »verdeckt« werden - man kann sich das 
vorstellen, als würde man eine Stelle eines Bildes untersuchen, indem man den Rest des Bildes mit Papier abdeckt.

Nur Kontext, der den jeweiligen Akteuren im Feld zu einem spezifischen Zeitpunkt zugänglich ist. Es geht hierbei um die Rekonstruktion dieses Wissens aus Sicht der Akteure, nicht aus der Vogelperspektive der Forschenden. Es ist daher nicht erlaubt, später im Video präsentierte Informationen zur Interpretation von früheren Zuständen heranzuziehen, da diese über das Wissen der Akteure zu einem bestimmten Zeitpunkt hinausgehen. Ein solches Vorgehen entspricht beispielsweise den Grundannahmen der Ethnomethodologie.

Alle fallinternen Informationen, also die Kontexte, die durch das Video selbst entstehen. Beispielsweise können Phänomene im Video durch später im Material auftauchende Informationen erklärt werden.

Alle fallinternen Informationen plus solche, die direkt mit der Entstehung des Videos verbunden sind, zum Beispiel Ankündigungstexte, Interviews, Produktionsmaterialien wie Skizzenbücher etc. Der Kontext wird hier erweitert, um eine multiperspektivische Analyse zu ermöglichen.

Das gesamte Weltwissen des/der Forschenden (z. B. figurale Hermeneutik, Grounded Theory).

Nicht nur das gesamte Weltwissen des einzelnen Forschenden, sondern auch das Weltwissen einer Gruppe von Forschenden. Auf dieses Verfahren setzt beispielsweise die Videografie (Tuma 2018).

Forschende sollten sich bewusst machen, welcher interpretatorische Rahmen den jeweiligen Zielen des konkreten Projekts entspricht.

Wie wird die Kamerahandlung analysiert?

Je nach verwendetem Ansatz sind unterschiedliche Videosorten die Grundlage der Analyse.

Videos, die wir im Netz finden oder die uns in anderer Form als fertige Produkte zugänglich werden, werfen ganz andere Fragen auf als solche, die unter einem forschenden Blick spezifisch für den Zweck der Forschung erstellt wurden. Bei bereits hergestellten Videos bildet neben dem Inhalt, also der Handlung vor der Kamera, auch die Kamerahandlung einen Schwerpunkt der Analyse, wobei Letztere neben der Aufnahme auch alle Handlungen der Postproduktion umfasst, also alle Entscheidungen 
über Einstellung, Quadrierung, Licht, Tiefenschärfe, Schnitt, Effekte usw.

Es ist immer zu klären, wie weit die Kamerahandlung Teil der Analyse ist. Entsprechend muss sie dann auch erfasst, transkribiert und analysiert werden. Bei spezifisch für die Forschung hergestellten Videos wird die Kamerahandlung mit der Intention der Wissensgenerierung vorgenommen und ist damit Teil der Methode, das heißt, sie ist auf ihre Geeignetheit für das Ziel der Forschung zu überprüfen und transparent zu machen. Je nach Fragestellung kann das eine oder das andere mehr gewichtet werden, es sollten jedoch immer beide Ebenen erfasst werden (Reichertz 2018).

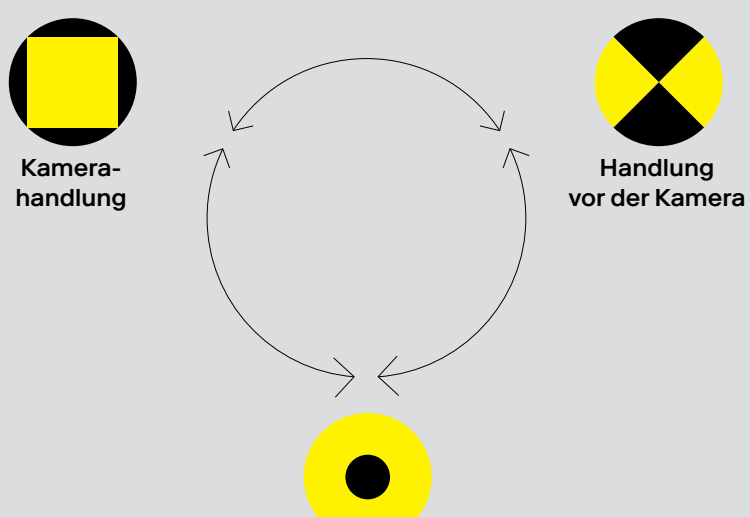

Analyse 
Wie wichtig sind der selektive und subjektive Blick?

Das Ideal einer »objektiven« Kamera strebt danach, die Vorselektion möglichst klein zu halten, ein breites Spektrum an Informationen einzufangen und einen weiten »Datenfokus « zu haben. Im Idealfall versucht die Kamera, »einfach alles « zu erfassen, was natürlich immer nur annäherungsweise gelingen kann. Durch das Aufstellen einer 360-Grad-Kamera kommen Forschende diesem Ideal zwar näher, als dies zuvor möglich war, aber auch hier finden sich Vorentscheidungen, beispielsweise durch den Aufstellungsort der Kamera. Im Gegensatz zur »objektiven« steht eine »subjektive Kamera«, die schon bei der Aufnahme klare Entscheidungen verlangt. Diese müssen oft unter einem künstlerisch-forschenden Blick sehr schnell und intuitiv getroffen werden. Die Videodaten sind dann selbstverständlich stark selegiert. Da die Annahme von Objektivität danach nicht mehr möglich ist, muss die aktive, subjektive Kamerahandlung Teil der Analyse werden. Man kann sich die AV-Datensorten als ein Kontinuum vorstellen:

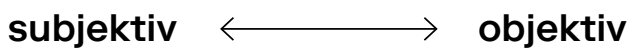

Dabei erfordert der Grad an Subjektivität jeweils andere Analysemethoden. Forschende müssen auch diese Entscheidung schon vor Beginn der Aufnahmen getroffen haben.

Top-down oder Bottom-up?

Neues Wissen entsteht immer in einem Zusammenspiel von theoretischen Konzepten und neu gewonnenen Daten, darin sind sich alle Forschungsansätze einig. Unterschiede gibt es aber in der Abfolge und Gewichtung: Was kommt zuerst? Was ist wichtiger?

Auch wenn die Begrifflichkeiten variieren, kann man unterscheiden zwischen Top-down-Ansätzen, die aus den theoretischen Konzepten Operationalisierungen ableiten, die sie dann an den Daten überprüfen, und Bottom-up-Ansätzen, die möglichst ohne Vorannahmen an die Daten herangehen, um dort emergierende Muster zu identifizieren. Die meisten Verfahren pendeln zwischen Bottom-up- und Top-down-Phasen hin und her (ältere, verwandte Begriffe für diese Phasen sind induktiv und deduktiv). 
Top-down-Verfahren ( $\rightarrow$ Abb. 4): Das theoretische Konzept, hier dargestellt als gelber Kreis mit schwarzem Punkt, wird zur Kategorienbildung verwendet, um die entsprechenden Phänomene dann in den Daten zu finden oder - wenn man sie nicht findet das Konzept zu hinterfragen.

Bottom-up-Verfahren ( $\rightarrow$ Abb. 5): Die Daten (Beobachtungen) sind primär und stehen am Anfang. Durch epistemische Praktiken werden Muster erkennbar - sie »emergieren«, kommen also an die Oberfläche, und werden erst danach theoretisch eingeordnet und versprachlicht.

\subsection{Die Analyse mit Videos und Annotationen}

Wenn die eigene Position und die Methode der Forschenden geklärt sind, werden die Daten entsprechend erhoben und aufbereitet und liegen danach in einer für das jeweilige Forschungsdesign geeigneten Form vor. Annotationen können schon bei der Erhebung der Videodaten als Live-Annotationen erfasst (beispielsweise mit dem Tool piecemaker2go) und später in beliebig vielen Analysedurchgängen mit Annotationen ergänzt werden, sodass etwas entsteht, was der Anthropologe Clifford Geertz als »Dichte Beschreibung « (thick description) bezeichnet hat (Geertz 1973). Nach unserem Modell in der Einleitung entspricht dies den »enriched data«.

Bereits beim Formulieren von Annotationen werden sich vorläufige Erkenntnisse einstellen, die wiederum notiert werden und das Material für jeweils weitere Analyseschritte darstellen. Insofern ist der Übergang von der Datenerhebung zur Datenanalyse oft fließend. Im Folgenden beschreiben wir die einzelnen Stufen als voneinander abgegrenzt, was sie in der Realität wohl eher selten sind.

\section{Beschreibung der Daten}

Der erste Schritt der Datenanalyse ist immer die Beschreibung der Daten im Überblick. Welche Datensorten liegen überhaupt vor, welchen Umfang und welche Qualität haben sie? In quantitativen Ansätzen benutzt man dafür die »deskriptive Statistik«, in qualitativen Ansätzen besteht dieser Schritt in einem Ausbreiten des gesamten Datenmaterials und einer textlichen Beschreibung der Datensorten, beispielsweise in Form einer Tabelle, 


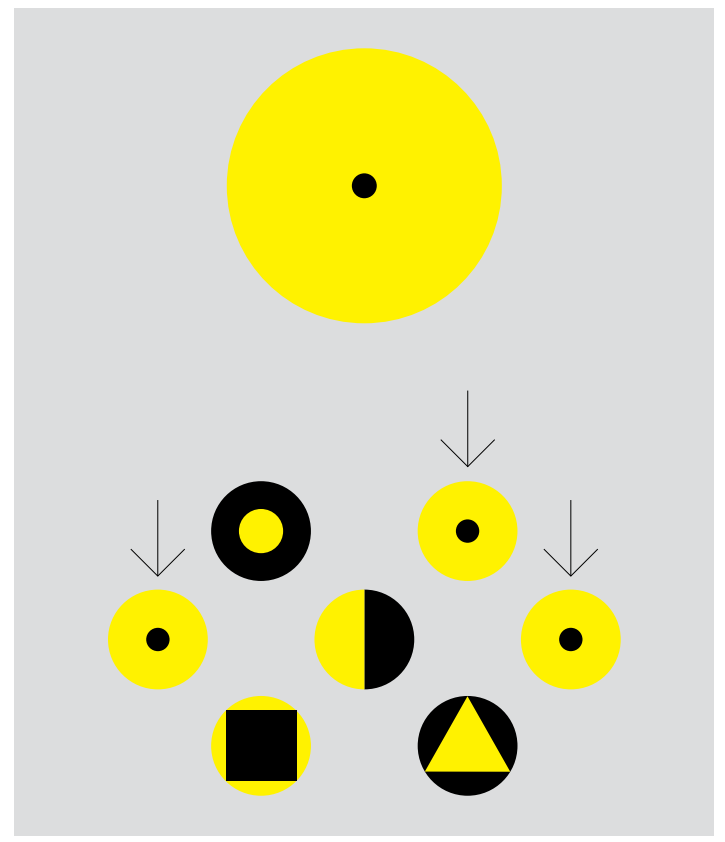

\section{4}

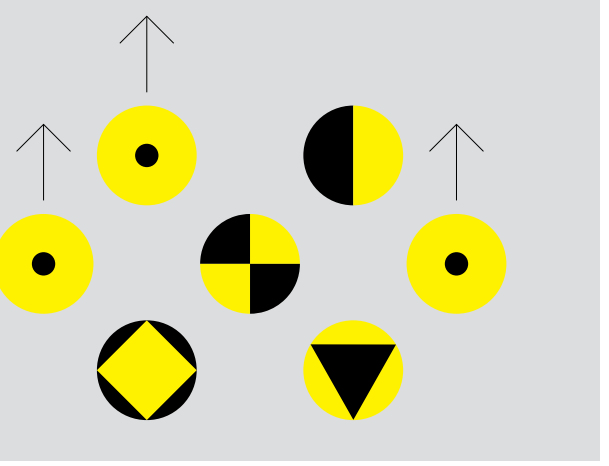

\section{5}

Abb. 04:

Top-down-Ansatz

(Quelle: eigene Darstellung)

Abb. 05:

Bottom-up-Ansatz

(Quelle. eigene Darstellung) 
von Feldnotizen oder eines Logbuchs. Die verschiedenen Videodaten werden hierbei benannt und einer ersten Durchsicht unterzogen.

Jürgen Raab und Marija Stanisavljevic empfehlen, das gesamte Material zunächst zu sichten, ohne sich Notizen zu machen. Erst nach diesem Gesamteindruck folgt ein zweiter Durchlauf mit Notizen (Raab/Stanisavljevic 2018). Andere Verfahren empfehlen ein Logbuch oder eine Grobkodierung als Liste oder Tabelle. Bei Videos sind für eine Sichtung des Materials ein gutes Ablagesystem der Videofiles und ein gutes System der Dateikennzeichnung absolut notwendig. Zur Beschreibung gehört auch eine kritische Bewertung der Daten.

Die Erstellung eines Überblicks zwingt nebenbei dazu, die Phase der Datengewinnung irgendwann abzuschließen: Ein Forschungsprojekt ist keine unendliche Reise, sondern bewegt sich auf ein Ende hin, weshalb die Übergänge von einer Phase in die nächste wirklich vollzogen werden sollten.

Definieren der Untersuchungseinheit (Unit of Analysis)

Was ist die kleinste Einheit, die untersucht werden soll, der »Unit of Analysis«? Man kann die Antwort meist aus der Theorie und dem Untersuchungskontext herleiten: Wie groß sind die zu untersuchenden Phänomene? Wenn wir kleine Fische fangen wollen, brauchen wir ein engmaschiges Netz, wenn wir große Fische fangen wollen, ein weitmaschiges Netz. Und wenn wir nicht wissen, was für Fische wir fangen wollen, sollten wir Proben nehmen und danach entscheiden. Mikrosoziologische Untersuchungen benötigen ganz andere Units of Analysis als beispielsweise soziologische Diskursanalysen. Ramey et al. weisen darauf hin, dass die Units of Analysis sich während des Forschungsprozesses ändern können, und plädieren für ein flexibles, iteratives Vorgehen (Ramey et al. 2016). Bei Videoanalysen kann die Untersuchungseinheit je nach Forschungsdesign stark variieren. 


\title{
Untersuchungseinheiten bei der Videoanalyse
}

\begin{abstract}
Sehr fein Die kleinste untersuchbare Einheit sind Stills des Videos, also Einzelbilder oder Frames. Diese Einheit wählen bildinterpretierende Verfahren, wie die figurative Hermeneutik, die sich ja von der Analyse unbewegter Bilder herleiten.
\end{abstract}

Fein

Hier sind die Untersuchungseinheiten Moves, das heißt kleinste bedeutungstragende Aktionen. Moves sind etwa vergleichbar mit Wörtern: Man kann sie einzeln interpretieren, weil jedes Wort eine Bedeutung trägt, aber sie sind Teile eines Interaktionsbeitrags. Ein Beispiel wäre eine Geste, die im Rahmen einer Äußerung gemacht wird, oder ein emotionaler Ausdruck, der diese begleitet. Solche Phänomene können auch unterschwellig im Mikrosekundenbereich auftreten. In diesem Fall werden sie erst durch das Video überhaupt beobachtbar, indem der Modus der Zeitlupe verwendet wird.

Mittel

Hier ist die Untersuchungseinheit ein einzelner Interaktionsbeitrag oder Turn, wobei die Teilnehmenden den Beginn und das Ende eines Turns selbst durch metapragmatische Signale markieren (Goffman 1974). Die Analyse vermeidet eine »künstliche« Zergliederung und folgt stattdessen dem »natürlichen« Verlauf des Geschehens (Interaktionsanalyse und Konversationsanalyse).

Personengroß

Hier liegt der Fokus auf individuellen Handlungseinheiten, die eine Strategie, einen Stil oder Habitus des jeweils einzelnen Interaktionsteilnehmers abbilden. Die Untersuchungseinheiten sind hier nicht auf die einzelne Interaktion, sondern auf die Person bezogen und finden sich daher über das Material verstreut, da, wo eine Äußerung »typisch« für diese Person erscheint. Solche Einheiten können über ihre Intentionalität oder Funktionalität beschrieben werden, beispielsweise eine Interaktion, wo A das Ziel hat, B auf eine bestimmte Weise zu belehren, was in der Analyse herausgearbeitet werden kann. Ein entsprechendes Konzept ist das des »Habitus« von Pierre Bourdieu.

Gesellschaftlich

Soziale Systeme oder Diskurse, also Abfolgen von Kommunikationen und Handlungen im öffentlichen Raum, die aufeinander Bezug nehmen, aber nicht Teil der unmittelbaren Interaktion sein müssen, also etwa diverse Kritiken eines Theaterstücks zu unterschiedlichen Zeiten und an unterschiedlichen Orten. Die Untersuchungseinheiten können in solchen Diskurs- und Viskursanalysen beliebig groß sein und eventuell sehr große Datenmengen umfassen. 
Indem im Research Video die Größe der Annotation auf dem Track sichtbar ist, liegt hier eine direkte Visualisierung des Unit of Analysis vor. Daher ist das Tool gut geeignet, Untersuchungseinheiten zu bestimmen. Sie bilden sich durch die Größe der Annotationen auf den Tracks ab. In einem Use Case wurden beispielsweise die Akte und Szenen eines Spielfilms mithilfe von Annotationen zergliedert $(\rightarrow$ Abb. 06).

Wie finde ich relevante Stellen im Material (Sampling)?

Nachdem die Daten beschrieben sind, müssen in der Regel kleinere Sequenzen identifiziert werden, die für die Analyse relevant sind. Dieser Vorgang, das Sampling, ist umso bedeutender, je stärker das Material selektiert wird. Es gibt mehrere anerkannte Verfahren.

\section{Methoden des Samplings}

Theoretisches Sampling
Die Selektion hat einen starken Bezug zur Forschungsfrage. Nur Sequenzen, die aufgrund theoretischer Überlegungen damit in Zusammenhang stehen, werden ausgewählt.
Whole-to-partMethode

Repräsentatives Sampling
Mehrere Betrachter schauen sich den gesamten Datencorpus an und suchen Übereinstimmung über die relevanten Stellen (Erickson 2006).
Selektion aufgrund von »Narrative Power«
Hier wird aus einer Menge ähnlicher Daten eine Sequenz ausgewählt, die »typisch« erscheint. Die Auswahl wird intersubjektiv validiert, indem man Experten befragt. werden diejenigen Clips gewählt, die eine Erzählung der Forschung unterstützen. Dabei wird die Geschichte mit einer »disciplined subjectivity« erzählt, die nicht vorgibt, objektive Perspektiven aufzunehmen, aber auch nicht rein fiktional ist. 
Einen Verfahrensvorschlag hierzu machen Raab und Stanisavljevic (2018):

○ gesamtes Material sichten, ohne Notizen zu machen

○ zweiter Durchlauf mit Notizen

$\circ$ Auswahl einer Stelle, die für die Fragestellung relevant sein könnte

○ Feinanalyse der Schlüsselstelle

$\circ$ Auswahl weiterer Stellen, die einen minimalen Kontrast bilden

$\circ$ Auswahl weiterer Stellen, die einen maximalen Kontrast bilden

Auf die Methode der minimalen/maximalen Kontrastbildung wird in Kapitel 8 noch näher eingegangen.

Tatsächlich gehört das Sampling zu den zentralen Praktiken qualitativer Forschung, auch wenn die Auswahl keineswegs methodologisch so präzise ist, wie dies gelegentlich behauptet wird. Im verbreiteten »Schneeballsystem « beispielsweise werden Ausgangsstellen einfach dadurch identifiziert, dass sie von mehreren Betrachtern übereinstimmend als »irgendwie interessant« bezeichnet werden. Die Auswahl der entsprechenden Sequenz bleibt daher trotz aller Methode bis zu einem gewissen Grad subjektiv und intuitiv.

Research Video bietet sehr gute Möglichkeiten, ein Video in Sequenzen zu zerlegen und diese zu visualisieren. Indem dasselbe Material von verschiedenen Forschenden gesampelt wird, kann eine gewisse intersubjektive Validierung schnell und anschaulich stattfinden. Damit ist das Tool eine gute Grundlage für das Sampling, unabhängig davon, welches Verfahren herangezogen wird.

\section{Transkription}

Zur Beschreibung der Daten gehört auch die Transkription als Beschreibung von beobachtbaren Phänomenen. Alle Forschungsansätze halten eine Transkription für notwendig, und es wurde vielfach darauf hingewiesen, dass jede Transkription bereits Vorentscheidungen enthält, die den Forschungsverlauf maßgeblich beeinflussen. "To create a transcript is to make consequential choices about which phenomena merit representation.« (Ramey 2016, S. 2) 
Die Transkription generiert dadurch eine »konzeptionelle Linse oder selektive Wahrnehmung, die gut reflektiert und begründet werden muss, weil sie bestimmt, was die Forschenden überhaupt wahrnehmen und was sie möglicherweise übersehen. Neben der Sprache wurde in Videos eine breite Palette von Modalitäten des Verhaltens transkribiert: Gesten, Blicke, Körperposition, Gesichtsausdruck, Bewegung, materielle Objekte u. a.

Die Forschungstradition und die Forschungsfrage legen fest, wie feinkörnig und wie multimodal eine Transkription zu sein hat - beispielsweise ob sie auch unbewusstes oder subliminales Verhalten wie Räuspern, Pausen, Schlucken, Fülllaute usw. enthalten soll. Ebenso entscheidet die Transkription darüber, wie die Kamerahandlung miteinbezogen werden soll.

Es existieren Systeme und Werkzeuge für die Transkription in den jeweiligen Forschungstraditionen (Moritz/Corsten 2018), die wie folgt eingeordnet werden können: »Instrumente wie HIAT, ELAN, ExMaralda sind für die eher sprechsprachorientierte Forschung geeignet. Für die sozialwissenschaftliche Analyse sind die Instrumente Feldpartitur, Moviscript oder TraVis geeignet. Weitere Softwaren wie Akira, atlas ti, MAXQDA oder NVIVO umgehen die Transkription und beschäftigen sich mit spezifischen Verfahren der Auswertung, etwa im Rahmen einer Qualitativen Inhaltsanalyse.«(Moritz in Moritz/Corsten 2018, S. 14). Ein Überblick über die einzelnen Tools findet sich bei Moritz (2014).

Annotierte Videos wie Research Video bieten sehr gute Möglichkeiten der Transkription, weil die jeweiligen Tracks beliebig gefüllt werden können. Es können also sowohl sprachliche also auch nonverbale Cues erfasst werden, außerdem auch Aspekte der Kamerahandlung. Die Forschenden können diese Modalitäten der Transkription auch während der Analyse noch erweitern.

\section{Datenanalyse - Muster erkennen}

Wissen manifestiert sich in Mustern und Zusammenhängen. Die epistemische (Erkenntnis ermöglichende) Funktion eines Videos wird dann deutlich, wenn es Möglichkeiten bietet, Daten neu zu sehen, neu zu sortieren, anders zu filtern und Muster zu erkennen, die dem bloßen Auge sonst verborgen bleiben würden. Die Analyse beginnt bei der konkreten Wahrnehmung und vertieft sich in immer weiteren Schritten zu komplexeren Verarbeitungen, genau wie zuerst das Auge die Wahrnehmungsdaten verarbeitet, 
danach der visuelle Kortex und danach andere Teile des Gehirns. In der künstlerischen Forschung ist dies nicht so sehr ein Prozess des Verifizierens oder Falsifizierens, sondern einer der Wahrnehmungsverstärkung.

Die erkannten Muster müssen nicht unbedingt kausale Muster sein, obwohl diese von der experimentellen Forschung bevorzugt werden, sondern können ebenso gut in phänomenologischen Muster bestehen, also in solchen, die es uns ermöglichen, einen Zusammenhang anders wahrzunehmen. Man kann sich das vorstellen wie die Kantendetektion beim Computer: Sie ermöglicht, unterschiedliche Flächen klar voneinander abzuheben, indem die Übergänge besonders hervorgehoben werden. Der Computer nimmt dafür einen Vergleich von Wahrnehmungspunkten mit ihrer direkten Umgebung vor und errechnet den Grad der Ähnlichkeit. Durch diese Methode sind Maschinen in der Lage, Umrisse wahrzunehmen und Objekte zu erkennen. Das menschliche Gehirn verfügt über einen ähnlichen Mechanismus, der die Kontraste dort, wo eine Fläche endet, verstärkt, sodass Objekte scharf aus dem Hintergrund hervortreten (Gestaltwahrnehmung).

Analog dazu kann die Analyse von Videomaterial über minimale-maximale Kontrastbildung genau solche Unterschiede und ihre exakten Verläufe herausarbeiten. Man kann diesen Vorgang auch mit dem Einbringen von Kontrastmitteln in der biologischen und medizinischen Forschung vergleichen: Durch bestimmte Praktiken werden die Daten so »eingefärbt«, dass Muster erkennbar werden ( $\rightarrow$ Abb. 07).

Durch das Verwenden von Tracks als Beobachtungskategorien können solche Muster im Research Video unmittelbar visualisiert werden. Blendet man einzelne Tracks aus, dann werden diese Muster noch deutlicher sichtbar. Sie werden dadurch nicht nur anschaulicher, sondern bleiben auch für andere Forschende nachvollziehbar, die den Schritt vom Videomaterial zu den Schlussfolgerungen zurückverfolgen wollen. Entsprechend bleibt die Interpretation eng am Material und kann von anderen Betrachtern hinterfragt werden: Sehen sie dasselbe Phänomen wie die Forschenden? Die Analyse bleibt dadurch »challengeable« und intersubjektiv validierbar. 


\section{Zum Weiterlesen}

Einen gut durchgearbeiteten Leitfaden haben Derry et al. (2010) entwickelt. Sie fokussieren auf vier Probleme: Selektion, Analyse, Technologie und Ethik (Derry et al. 2010). Eine weitere nützliche Quelle ist der Bericht von Ramey et al. (2016) zu einem Symposium über Standards und Heuristiken der Videoanalyse, der Faustregeln für die wichtigsten Probleme formuliert.

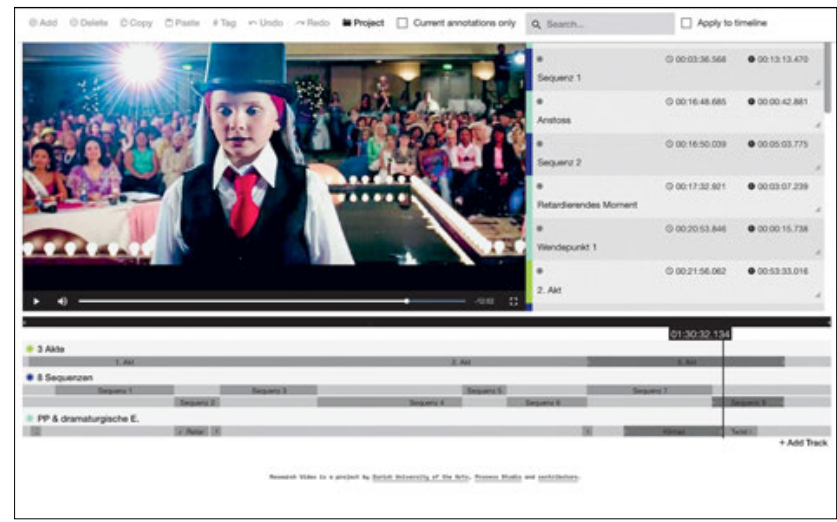

\section{6}

\section{0:00:00.000}

๑) Introduction \& Method

Me 0

Intr

๑ Sequence Description
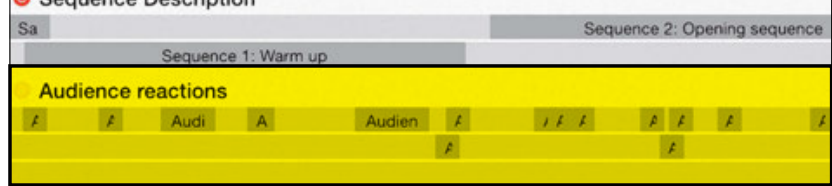

Conclusions

References

Analysis Warm up

L E

$+$

Abb. 06:

Sequenzbildung mit Tracks und

Annotationen

Abb. 07:

Beispielprojekt. Die Annotationen

zeigen den Rhythmus der Zuschauer-

reaktionen. 


\section{Die erkannten Muster müsse} Muster sein, obwohl diese vo Forschung bevorzugt werder gut in phänomenologischen I solchen, die es uns ermöglich anders wahrzunehmen. 
n nicht unbedingt kausale n der experimentellen , sondern können ebenso Muster bestehen, also in ien, einen Zusammenhang 